\title{
Quality of Life in a Randomized Phase II Study to Determine the Optimal Dose of 3-Week Cycle Nab- Paclitaxel in Patients with Metastatic Breast Cancer
}

Naruto Taira ( $\sim$ ntaira@md.okayama-u.ac.jp )

Okayama University Hospital: Okayama Daigaku Byoin https://orcid.org/0000-0003-4266-1117

Kosuke Kashiwabara

University of Tokyo Hospital: Tokyo Daigaku Igakubu Fuzoku Byoin

Junji Tsurutani

Showa University: Showa Daigaku

Masahiro Kitada

Asahikawa Medical University: Asahikawa Ika Daigaku

Masato Takahashi

National Hospital Organization Hokkaido Cancer Center

Yuichiro Kikawa

Kansai Medical University: Kansai Ika Daigaku

Eiko Sakata

Niigata City General Hospital: Niigata Shimin Byoin

Yoichi Naito

National Cancer Center-Hospital East: Kokuritsu Gan Center Higashi Byoin

Yoshie Hasegawa

Hirosaki Municipal Hospital

Tsuyoshi Saito

Japanese Red Cross Saitama Hospital

\section{Tsutomu Iwasa}

Kindai University Faculty of Medicine Graduate School of Medical Sciences: Kinki Daigaku Igakubu

Daigakuin Igaku Kenkyuka

\section{Tsutomu Takashima}

Osaka University School of Medicine Graduate School of Medicine: Osaka Daigaku Daigakuin Igakukei

Kenkyuka Igakubu

Tomohiko Aihara

Aihara Hospital

Hirofumi Mukai

National Cancer Center-Hospital East: Kokuritsu Gan Center Higashi Byoin

Fumikata Hara 


\section{Research Article}

Keywords: metastatic breast cancer, chemotherapy, nab-paclitaxel, QoL, CIPN, fatigue

Posted Date: February 8th, 2021

DOl: https://doi.org/10.21203/rs.3.rs-178724/v1

License: (c) (i) This work is licensed under a Creative Commons Attribution 4.0 International License. Read Full License 


\section{Abstract}

Purpose: To report our findings on quality of life (QoL) in a randomized phase II study to determine the optimal dose of 3-week cycle nab-paclitaxel (q3w nab-PTX) in patients with metastatic breast cancer $(\mathrm{MBC})$.

Materials and Methods: Patients with HER2-negative MBC were randomly assigned to three different doses of q3w nab-PTX (SD: $260 \mathrm{mg} / \mathrm{m}^{2}$ vs. MD: $220 \mathrm{mg} / \mathrm{m}^{2}$ vs. LD: $180 \mathrm{mg} / \mathrm{m}^{2}$ ). QoL was assessed at baseline and during the $2 \mathrm{nd}$, 4th and 6 th courses of treatment using the Functional Assessment of Cancer Therapy-Taxane (FACT-Taxane), Cancer Fatigue Scale (CFS) and EuroQol 5-Dimension (EQ-5D). Comparisons were performed with mixed-model repeated-measures (MMRM).

Results: A total of 141 patients were enrolled in the parent study, and $136(96 \%)(44,45$ and 47 in the SD, $M D$, and LD groups) were included in the analysis. MMRM analysis showed that the difference from the baseline FACT-Taxane trial outcome index at MD and LD were significantly higher than that at SD (MD vs. SD $p<0.001$, LD vs. SD $p<0.001)$. Differences from baseline for FACT-Taxane total, physical and emotional well-being, and taxane subscale scores at MD and LD were also higher than at SD. The difference from baseline for the CFS score at LD was lower than at SD $(p=0.013)$ and those for EQ-5D utility scores at MD and LD were higher than at SD (MD vs. SD $p=0.011$, LD vs. SD $p<0.001$ ).

Conclusion: QoL of patients treated with 220 or $180 \mathrm{mg} / \mathrm{m}^{2}$ of q3w nab-PTX was significantly better than that of patients treated with $260 \mathrm{mg} / \mathrm{m}^{2}$.

\section{Introduction}

Nab-paclitaxel (PTX) is a chemotherapeutic agent for metastatic breast cancer that is administered as a nanoparticulate preparation of PTX bound to human serum albumin. Unlike conventional, solvent-based PTX (so-PTX), polyoxyethylene castor oil or absolute ethanol are not used as a solvent. For this reason, pre-medication with steroid hormones is not needed and a high dose of PTX can be administered in a short time. In patients with metastatic breast cancer, nab-PTX $260 \mathrm{mg} / \mathrm{m}^{2}$ every 3 weeks (q3w) was found to be superior for response rate (RR) and progression-free survival (PFS) compared to q3w so-PTX $175 \mathrm{mg} / \mathrm{m}^{2}$ [1]. However, in the nab-PTX group, the dose of PTX was approximately 1.5 times higher and the frequency of chemotherapy-induced peripheral neuropathy (CIPN) was significantly higher.

In Japan, nab-PTX was approved in 2010 for treatment of metastatic breast cancer. The recommended dosage of nab-PTX by the Pharmaceuticals and Medical Devices Agency is $260 \mathrm{mg} / \mathrm{m}^{2}$ intravenously over 30 minutes every 3 weeks for metastatic breast cancer. However, a survey on actual use started after release of nab-PTX found that about one-third of cases were treated with a reduced initial dose [2]. In addition, about $30 \%$ of cases that started with the standard dose required dose reduction, mainly due to myelosuppression and CIPN. In particular, the rate of CIPN of Grade $\geq 2$ was as high as about $40 \%$. Based 
on these real-world findings, the current standard dose of nab-PTX is highly toxic and may be problematic over time. Thus, the current study was planned to investigate the optimal dose of nab-PTX.

This study compared three different doses of q3w nab-PTX (SD: $260 \mathrm{mg} / \mathrm{m}^{2} \mathrm{vs}$. MD: $220 \mathrm{mg} / \mathrm{m}^{2} \mathrm{vs}$. LD: $180 \mathrm{mg} / \mathrm{m}^{2}$ ) in patients with human epidermal growth factor receptor type2 (HER2) negative metastatic breast cancer [3]. The primary endpoint was PFS. Grade 3/4 neuropathy rates at the three doses were estimated by logistic regression. The optimal dose was defined by 2-step selection. First, if the hazard ratio (HR) for PFS was $<0.75$ or $>1.33$, the inferior dose was dropped. Then, if the estimated incidence rate of grade $3 / 4$ neuropathy was $>10 \%$, the other dose was dropped. Primary analysis showed that lowdose nab-PTX at $180 \mathrm{mg} / \mathrm{m}^{2} \mathrm{q} 3 \mathrm{w}$ may be the optimal therapy with meaningful efficacy and favorable toxicity in patients with metastatic breast cancer [4].

In general, the frequency and severity of adverse events caused by chemotherapy are dose-dependent, so a potential benefit of using low-dose nab-PTX is reduction of adverse events. The frequency or severity of CIPN is likely to be reduced with a low-dose nab-PTX regimen, and this dose may also reduce symptoms such as fatigue caused by cancer chemotherapy. Based on the hypothesis that low-dose nab-PTX treatment will result in better quality of life (QoL) than standard dose nab-PTX, the purpose of this QoL substudy is to show the superiority of low dose PTX in terms of QoL.

\section{Materials And Methods Study design}

This study is a multicenter, open-label, phase 2 randomized controlled trial (RCT) conducted in Japan. Details of study protocols have been reported elsewhere [3]. Briefly, the inclusion criteria were histologically diagnosed invasive breast cancer; incurable metastatic disease; aged 20-75 years; an Eastern Cooperative Oncology Group performance status (PS) score of 0 or 1 ; and chemotherapy for metastatic breast cancer allowed up to one regimen. The exclusion criteria were HER2-positive breast cancer; patients with CIPN of grade $\geq 2$; and brain metastases with symptoms or requiring treatment.

Eligible patients were randomly assigned in a 1:1:1 ratio to receive any of 3 doses of nab-PTX: standard dose (SD) $260 \mathrm{mg} / \mathrm{m}^{2}$, medium dose (MD) $220 \mathrm{mg} / \mathrm{m}^{2}$, and low dose (LD) $180 \mathrm{mg} / \mathrm{m}^{2}$. Stratification factors were a history of chemotherapy for metastatic disease (yes vs. no), hormone receptor status (positive vs. negative), history of taxane treatment including perioperative use (yes vs. no), relapse-free interval ( $<2$ vs. $\geq 2$ years), and participating institution.

The patient received the assigned dose of nab-PTX once every 3 weeks for 30 min until disease progression or adverse events prevented continued treatment. After starting the protocol treatment, if a patient developed grade 4 thrombocytopenia, grade 3 thrombocytopenia requiring blood transfusion, neutrophils $<500 / \mathrm{mm}^{3}$ lasting for $\geq 7$ days, or grade $\geq 3$ non-hematological toxicity (excluding nausea, 
vomiting, and diarrhea), the dose in the next course was reduced by one step to 220,180 and $140 \mathrm{mg} / \mathrm{m}^{2}$ for the SD, MD and LD groups, respectively.

All subjects gave written informed consent before enrollment in the study. The study was approved by the institutional ethics committee on human research at all participating medical institutions.

\section{QoL assessment}

QoL of the study population was assessed using the Japanese version of the Functional Assessment of Cancer Therapy-Taxane (FACT-Taxane) scale [5]. FACT-Taxane was developed to measure adverse events and QoL in patients undergoing taxane treatment. It consists of a 27-item general cancer QoL measure [7 for physical well-being (PWB), 7 for social and family well-being (SFWB), 6 for emotional well-being (EWB), and 7 for functional well-being (FWB)] and a 16-item taxane subscale, including an 11-item neurotoxicity subscale and 5 questions on symptoms related to arthralgia, myalgia, and skin discoloration. Each FACT-Taxane item or question has response choices from 0 ("not at all") to 4 ("very much"). Scores range from 0 to 172 and a higher score indicates better QoL. The Japanese version of EuroQol 5-dimension (EQ-5D)-3L was used for preference-based measures of QoL, and was scored using a conversion table for the Japanese population $[6,7]$.

Fatigue in cancer patients was assessed using the Cancer Fatigue Scale (CFS) [8]. The CFS was developed to measure fatigue in cancer patients in Japan, with 15 items in 3 subscales: 7 for physical (score range $0-28), 4$ for affective $(0-16)$, and 4 for cognitive $(0-16)$ subscales. Comprehensive fatigue in cancer patients is calculated as the sum of the three domains. The score is $0-60$, and a higher score indicates that fatigue is noticeable.

All patients in the parent trial were invited to be a part of the QoL substudy. Self-administered questionnaires were distributed by medical staff to subjects and returned to the data center by mail. The date of the investigation and the allowed survey period were at registration (study entry to the start of protocol treatment), and during the $2 \mathrm{nd}$, 4th and 6th courses of protocol treatment. If treatment was discontinued due to disease progression, complications, exacerbations, or adverse events, QoL evaluation was discontinued.

\section{Analysis}

The target group for the QoL substudy analysis included all registered cases except those that 1) did not meet the eligibility criteria. 2) for whom no protocol treatment was performed, or 3) QoL evaluation was not performed before the start of protocol treatment. The response rate was defined as the number of responses collected/the number of expected responses. All questionnaires were quantified using a scoring manual, and basic statistics for each group and each survey point were calculated.

The hypothesis for QoL evaluation was that QoL at MD or LD would be better than at SD. The primary outcome of the QoL substudy was the FACT-Taxane Trial Outcome Index (TOI) in the 2nd, 4th, and 6th 
courses of protocol treatment. FACT-Taxane TOI is a 40-item aggregate of the PWB, FWB, and taxane subscale, and is often used as a single clinical trial outcome index.

For comparison of scores calculated as continuous variables, analysis was performed using a mixed effects model for repeated measures (MMRM). The model used the scores obtained at each survey point, except baseline, as an outcome, and the baseline value, group, survey point, and interaction terms of group and survey point as explanatory variables. The least-squares mean square value at each survey point for each group with the $95 \%$ confidence interval $(\mathrm{Cl})$, the intergroup difference at each survey point with the $95 \% \mathrm{Cl}$, and the $p$ value were calculated.

Missing data were not imputed. There were no multiple-comparison adjustments. All tests were two-sided, and $p<0.05$ was considered to be significant. SAS v. 9.3 was used for analysis.

\section{Results}

\section{Patients and characteristics}

From February 2015 through June 2018, a total of 141 eligible patients were enrolled in the parent study, and 136 patients (96\%) (44, 45 and 47 in the SD, MD, and LD groups, respectively) were included in QoL analysis (Fig. 1).

The characteristics of the patients in the QoL substudy are shown in Table 1. In the SD, MD and LD groups, the mean ages were 57.9, 58.4 and 57.6 years, and the mean body mass indexes (BMIs) were $25.2,23.6$ and $22.3 \mathrm{~kg} / \mathrm{m}^{2}$, respectively, with significant differences among BMls $(p=0.03)$. There was no significant difference in PS, estrogen receptor status, pre-treatment by chemotherapy, pre-treatment by taxane, and comorbidities.

The response rates of questionnaire in the $2 \mathrm{nd}$, 4th, and 6 th courses of protocol treatment were $98 \%, 95 \%$ and $97 \%$ in the SD group, $98 \%, 100 \%$ and $97 \%$ in the MD group, and $91 \%, 97 \%$ and $94 \%$ in the LD group, respectively. Reasons for missing data are listed in Appendix Table A1.

\section{Taxane-related QoL}

Mean baseline scores and standard deviations for FACT-Taxane and the Taxane subscale are shown in Table 2. These scores did not differ significantly among the groups at baseline. In comparison of baseline-adjusted means by MMRM analysis, there were significant differences between SD and MD for FACT-Taxane TOI $(p<0.001)$, FACT-Taxane total $(p<0.001)$, Taxane subscale $(p<0.001)$ (Fig. 2$),$ PWB $(p$ $=0.002)$, SWB $(p=0.037)$, and EWB $(p=0.024)$; and between SD and LD for FACT-Taxane TOI $(p<0.001)$, FACT-Taxane total $(p<0.001)$, FACT-General total $(p=0.011)$, Taxane subscale $(p<0.001)$ (Fig. 2$)$, PWB $(p$ $<0.001)$ and EWB $(p=0.004)$. The mean change from baseline and $95 \% \mathrm{Cl}$ for each dose are shown in Appendix Figure A1. Overall, taxane-related QoL was better maintained at MD and LD than at SD. There were no significant differences among the doses in EQ-5D utility scores at baseline (Table 2). In a 
comparison of baseline adjusted means by MMRM analysis, there were significant differences in EQ-5D utility scores between SD and MD ( $p=0.011)$, and SD and LD $(p<0.001)$ (Fig. 3$)$.

\section{Cancer fatigue}

There were no significant differences among the groups in CFS total score, and physical, affective and cognitive subscores at baseline (Table 2). In comparison of baseline-adjusted means by MMRM analysis, these variables also showed no significant differences at SD and MD (Fig. 4). However, there were significant differences between SD and LD for CFS total score $(p=0.013)$, and physical $(p=0.001)$ and cognitive ( $p=0.033$ ) subscores (Fig. 4). The mean change from baseline and $95 \% \mathrm{Cl}$ for each dose are shown in Appendix Figure A2.

\section{Discussion}

We found that the initial dose of q3w nab-PTX had a significant effect on taxane-related QoL and cancer fatigue. QoL of patients with metastatic breast cancer treated with $260 \mathrm{mg} / \mathrm{m}^{2}$ of q3w nab-PTX was significantly worse compared to those treated with $220 \mathrm{mg} / \mathrm{m}^{2}$ or $180 \mathrm{mg} / \mathrm{m}^{2}$ of q3w nab-PTX. Primary endpoint analysis showed that intravenous administration of low-dose nab-PTX at $180 \mathrm{mg} / \mathrm{m}^{2} \mathrm{q} 3 \mathrm{w}$ may be the optimal therapy with meaningful efficacy and favorable toxicity for patients with metastatic breast cancer [4]. The QoL substudy also showed that low-dose nab-PTX at $180 \mathrm{mg} / \mathrm{m}^{2} \mathrm{q} 3 \mathrm{w}$ is optimal in terms of QoL and cancer-related fatigue.

Standard doses of chemotherapeutic agents should be determined based on risk and benefit balance. However, in terms of dose intensity, the standard dose is often determined by acute toxicities such as hematologic toxicity, diarrhea, and vomiting during development of the drug. Regarding the cumulative toxicity of chemotherapy, a strategy of continuing treatment by short-term drug withdrawal or reducing the dose is used based on observation of adverse events. However, some adverse effects of chemotherapy are not easily improved by withdrawing or reducing the dose of chemotherapy, and CIPN is an example. In a discussion at the start of the study, treatment with nab-PTX using drug reduction and withdrawal was suggested to be optimal. However, we obtained a result that overturned this opinion, since we found that the initial dose of nab-PTX has a significant effect on subsequent patient QoL.

CIPN is a major clinical challenge due to lack of effective treatment and impact on QoL [9]. There are no drugs for preventing or ameliorating CIPN in a long-term course, and chemotherapy schedule modification is often required to limit its severity; however, this also may limit the efficacy of the cancer treatment. Moreover, symptomatic therapy is often ineffective in reducing CIPN symptoms [10]. Taxanes are representative chemotherapeutic agents that induce CIPN. Taxanes inhibit depolymerization of microtubules [11, 12], and CIPN may be caused by the resulting abnormal aggregation of microtubules in neuronal cells. Polyoxyethylated castor oil, which is used as a vehicle for taxanes, may also increase the risk for prolonged peripheral neuropathy [13]. Animal studies have shown that the time to recovery from 
CIPN depends on the drug, and that the morphological effect on axons in PTX-treated animals is long lasting [14].

Nab-PTX is an albumin-bound, nanoparticle formulation of PTX that was developed to reduce the incidence of CIPN compared to that with conventional taxanes [15, 16]. However, in the CA012-0 trial, a phase III study comparing 3-week cycles of nab-PTX $\left(260 \mathrm{mg} / \mathrm{m}^{2}\right)$ and so-PTX $\left(175 \mathrm{mg} / \mathrm{m}^{2}\right)$ in patients with metastatic breast cancer, CIPN occurred more frequently with nab-PTX, despite nab-PTX showing greater efficacy in terms of RR [1]. In contrast, superiority of nab-PTX over so-PTX in PFS was not found in patients with metastatic breast cancer in the CALGB40502 phase III trial, and hematologic toxicity of grade $\geq 3$ and CIPN occurred more frequently with nab-PTX [17]. Dose reduction was more common and occurred earlier with nab-PTX, and discontinuation rates were also higher. Therefore, it was concluded that the dose of nab-PTX used in the study $\left(150 \mathrm{mg} / \mathrm{m}^{2} /\right.$ week $)$ was not appropriate and resulted in significant toxicity. These studies indicate that the initial dose of nab-PTX can cause adverse events that may have a long recovery time and a significant effect on patient outcomes, consistent with the findings obtained in this study.

Cancer-related fatigue causes disruption of all aspects of QoL and may be a risk factor for reduced survival [18]. Fatigue may be elevated before treatment onset and typically increases during cancer treatment, including that with radiation, chemotherapy, hormonal, and/or biological therapies. The rate of cancer-related fatigue ranges from 4-91\%, depending on the population studied and the methods of assessment [19]. In a RCT comparing taxanes and S-1 in patients with metastatic breast cancer in Japan, the frequency of fatigue in the taxane group was approximately $50 \%$ in all grades [20]. However, these data are based on physician's assessments, which may be underreported, and the actual rate of fatigue in taxane-based chemotherapy for metastatic breast cancer is likely to be higher [21]. Fatigue in cancer patients is multifactorial and may be influenced by demographic, medical, psychosocial, behavioral, and biological factors [18]. Nevertheless, the initial dose of nab-PTX had a significant effect on subjective fatigue in this study, especially for physical fatigue. At the current standard dose of $260 \mathrm{mg} / \mathrm{m}^{2}$, physical fatigue worsened over the course of treatment, but no worsening tendency was observed at 220 or 180 $\mathrm{mg} / \mathrm{m}^{2}$.

Both CIPN and fatigue are symptoms that are difficult for physicians to evaluate accurately, and in clinical trials, the discrepancy between physicians' assessments and patients reported outcomes (PROs) can be a problem [21]. In a RCT of adjuvant chemotherapy with taxanes for breast cancer, a substudy of the agreement between physician and patient assessments for CIPN gave kappa coefficients for sensory and motor symptoms of 0.16 and 0.22 , which are extremely low [22]. In a comparative study of clinician and patient assessment of symptoms in lung cancer patients, $41 \%$ of clinicians underreported symptoms of fatigue compared to patient reports [23]. Therefore, as the results of this study indicate, there is a limit to the therapeutic strategy of monitoring the cumulative toxicity associated with chemotherapy by physician assessment and reducing the dose or withdrawing the drug when this toxicity becomes significant. If a treatment strategy is to truly maintain and improve QoL, it is essential to monitor PROs in 
daily clinical practice [24]. It is also important to verify the dose that achieves the optimal risk and benefit balance after marketing of a new chemotherapeutic agent, particularly for drugs with cumulative toxicity.

A limitation of this study is that it is based on a secondary endpoint of a small randomized phase 2 trial, with no adjustment for multiple tests. Also, a relatively short-term evaluation up to the sixth cycle was used, and long-term effects were not examined. Furthermore, a weekly schedule of nab-PTX is more commonly used to treat patients with breast cancer, and the significance of our findings for the reduced dose of q3w nab-PTX may be limited. Nab-PTX at $100 \mathrm{mg} / \mathrm{m}^{2}$ can be administered weekly to reduce the incidence and degree of myalgia. Nonetheless, it was not until recently that the weekly nab-PTX regimen was adopted in Japan for treatment of patients with metastatic breast cancer due to previous failures to demonstrate its superiority to q3w docetaxel in terms of PFS [25]. There should be room for less frequent regimens, especially in patients with limited access to clinics or in those who might benefit from minimizing the risk of coronavirus infection during the COVID-19 pandemic.

We conclude that the initial dose of nab-PTX affects subsequent taxane-related QoL, as well as cancerrelated fatigue. Low dose nab-paclitaxel at $180 \mathrm{mg} / \mathrm{m}^{2} \mathrm{q} 3 \mathrm{w}$ may be an optimal dose in terms of PFS, taxane-related QoL and cancer-related fatigue. Further evaluation is warranted to confirm these findings in a larger trial.

\section{Declarations}

\section{Funding}

This study was sponsored by the Comprehensive Support Project for Oncology Research in Breast Cancer, with research funds provided by Taiho Pharmaceutical under the study contract. Taiho Pharmaceutical took no part in this study other than providing information relevant to proper use of the study drug.

\section{Conflict of Interest}

Junji Tsurutani received research fund from Daiichi Sankyo; honorarium from Novartis, Taiho, Eisai, Chugai, and Kyowa Kirin; personal fees for participating in advisory boards for Eisai and Asahi Kasei; and support for travel expenses from Daiichi Sankyo.

Masato Takahashi received honorarium for lecture from Taiho.

Yoichi Naito received research fund from Roche Diagnostics; and honorarium for lecture from Roche Diagnostics, Novartis, Eisai, Chugai Pharmaceutical, Fuji Film Toyama Chemistry, and Taiho.

Hirofumi Mukai received honorarium from Pfizer, Takeda, Daiichi Sankyo and Taiho; and research grant from Japanese government, Daiichi Sankyo, and Pfizer.

Hirofumi Mukai is a member of Board of Directors of Japan Breast Cancer Society. 
Acknowledgments

We thank all the patients and their families for taking part in the study. We are also grateful to Miss $\mathrm{H}$. Shinano for the administrative support required to carry out this research.

\section{Clinical Trial Registration}

The protocol was registered at the website of the University Hospital Medical Information Network (UMIN), Japan (protocol ID: UMIN000015516), on November 1, 2014. Details are available at the following address:

https://upload.umin.ac.jp/cgi-open-bin/ctr_e/ctr_view.cgi?recptno=R000017916

\section{Ethics Approval}

This study was reviewed and approved by independent ethics committees and institutional review boards. Written informed consent was obtained from all patients.

\section{Data Sharing}

Due to the nature of this research, participants in the study did not agree for their data to be shared publicly, so supporting data is not available.

\section{Authors' Contributions}

Conceptualization: Fumikata Hara, Hirofumi Mukai and Naruto Taira; Methodology: Fumikata Hara, Kosuke Kashiwabara, Tsuyoshi Saito, Naruto Taira, Tsutomu Takashima and Junji Tsurutani; Software and formal analysis: Kosuke Kashiwabara; Investigation: Naruto Taira, Junji Tsurutani, Masahiro Kitada, Masato Takahashi, Yuichiro Kikawa, Eiko Sakata, Yoichi Naito, Yoshie Hasegawa, Tsuyoshi Saito, Tsutomu Iwasa, Tsutomu Takashima, Tomohiko Aihara, Hirofumi Mukai and Fumikata Hara; Data curation: Tomohiko Aihara; Writing-original draft: Naruto Taira, Kosuke Kashiwabara and Fumikata Hara; Writing-review and editing: all authors; Visualization: Naruto Taira, Kosuke Kashiwabara and Fumikata Hara; Supervision: Hirofumi Mukai; Data curation: Fumikata Hara and Kosuke Kashiwabara; Project administration: Fumikata Hara; Funding acquisition: Fumikata Hara and Hirofumi Mukai.

\section{References}

1. Gradishar WJ, Tjulandin S, Davidson N, Shaw H, Desai N, Bhar P et al (2005) Phase III trial of nanoparticle albumin-bound paclitaxel compared with polyethylated castor oil-based paclitaxel in women with breast cancer. J Clin Oncol 31:7794-7803

2. Nakamura S, Iwata H, Funato Y, Ito K, Ito $Y$ (2015) [Results of a drug use investigation of nanoparticle albumin-bound paclitaxel for breast cancer]. Gan To Kagaku Ryoho 42:447-455 
3. Hara F, Takashima T, Tsurutani J, Saito T, Taira N, Kashiwabara K et al (2016) Randomized, optimal dose finding, phase II study of tri-weekly nab-paclitaxel in patients with metastatic breast cancer (ABROAD). J Clin Trials 6:267

4. Tsurutani J, Hara F, Kitada M, Takahashi M, Kikawa Y, Kato H et al (2020) Randomized phase II study to determine the optimal dose of 3-week cycle nab-paclitaxel in patients with metastatic breast cancer. Breast 55:63-68

5. Cella D, Peterman A, Hudgens S, Webster K, Socinski MA (2003) Measuring the side effects of taxane therapy in oncology: the functional assesment of cancer therapy-taxane (FACT-taxane). Cancer 98:822-831

6. Brooks R (1996) EuroQol: the current state of play. Health Policy 37:58-72

7. Tsuchiya A, Ikeda S, Ikegami N, Nishimura S, Sakai I, Fukuda T et al (2002) Estimating an EQ-5D population value set: the case of Japan. Health Econ 11:341-353

8. Okuyama T, Akechi T, Kugaya A, Okamura H, Shima Y, Maruguchi M et al (2000) Development and validation of the Cancer Fatigue Scale: a brief, three-dimensional, self-ratingscale for assessment of fatigue in cancer patients. J Pain Symptom Manage 19:5-14

9. Mols F, Beijers T, Vreugdenhil G, van de Poll-Franse L (2014) Chemotherapy-induced peripheral neuropathy and its association with quality of life: a systematic review. Support Care Cancer 22:2261-2269

10. Cavaletti G, Marmiroli P (2010) Chemotherapy-induced peripheral neurotoxicity. Nat Rev Neurol 6:657-666

11. Parness J, Horwitz SB (1981) Taxol binds to polymerized tubulin in vitro. J Cell Biol 91:479-487

12. Schiff PB, Fant J, Horwitz SB (1979) Promotion of microtubule assembly in vitro by taxol. Nature 277:665-667

13. Roytta M, Raine CS (1985) Taxol-induced neuropathy: further ultrastructural studies of nerve fibre changes in situ. J Neurocytol 14:157-175

14. Cook BM, Wozniak KM, Proctor DA, Bromberg RB, Wu Y, Slusher BS et al (2018) Differential morphological and biochemical recovery from chemotherapy-induced peripheral neuropathy following paclitaxel, ixabepilone, or eribulin treatment in mouse sciatic nerves. Neurotox Res 34:677692

15. Yardley DA (2013) nab-Paclitaxel mechanisms of action and delivery. J Control Release 170:365372

16. Desai N, Trieu V, Yao Z, Louie L, Ci S, Yang A et al (2006) Increased antitumor activity, intratumor paclitaxel concentrations, and endothelial cell transport of cremophor-free, albumin-bound paclitaxel, ABI-007, compared with cremophor-based paclitaxel. Clin Cancer Res 12:1317-1324

17. Rugo HS, Barry WT, Moreno-Aspitia A, Lyss AP, Cirrincione C, Leung E et al (2015) Randomized phase III trial of paclitaxel once per week compared with nanoparticle albumin-bound nab-paclitaxel once per week or ixabepilone with bevacizumab as first-line chemotherapy for locally recurrent or metastatic breast cancer: CALGB 40502/NCCTG N063H (Alliance). J Clin Oncol 33:2361-2369 
18. Bower JE (2014) Cancer-related fatigue: mechanisms, risk factors, and treatments. Nat Rev Clin Oncol 11:597-609

19. Lawrence DP, Kupelnick B, Miller K, Devine D, Lau J (2004) Evidence report on the occurrence, assessment, and treatment of fatigue in cancer patients. J Natl Cancer Inst Monogr 32:40-50

20. Takashima T, Mukai H, Hara F, Matsubara N, Saito T, Takano T et al, SELECT BC Study Group (2015) Taxanes versus S-1 as the first-line chemotherapy for metastatic breast cancer (SELECT BC): an open-label, non-inferiority, randomised phase 3 trial. Lancet Oncol 17:90-98

21. Seruga B, Templeton AJ, Badillo FE, Ocana A, Amir E, Tannock IF (2016) Under-reporting of harm in clinical trials. Lancet Oncol 17:e209-e219

22. Shimozuma K, Ohashi Y, Takeuchi A, Aranishi T, Morita S, Kuroi K et al (2019) Feasibility and validity of the Patient Neurotoxicity Questionnaire during taxane chemotherapy in a phase III randomized trial in patients with breast cancer: N-SAS BC 02. Support Care Cancer 17:1483-1491

23. Basch E, lasonos A, McDonough T, Barz A, Culkin A, Kris MG et al (2006) Patient versus clinician symptom reporting using the National Cancer Institute Common Terminology Criteria for Adverse Events: results of a questionnaire-based study. Lancet Oncol 7:903-909

24. LeBlanc TW, Abernethy AP (2017) Patient-reported outcomes in cancer care: hearing the patient voice at greater volume. Nat Rev Clin Oncol 14:763-772

25. Tamura K, Inoue K, Masuda N, Takao S, Kashiwaba M, Tokuda Y et al (2017) Randomized phase II study of nab-paclitaxel as first-line chemotherapy in patients with HER2-negative metastatic breast cancer. Cancer Sci 108:987-994

\section{Figures}




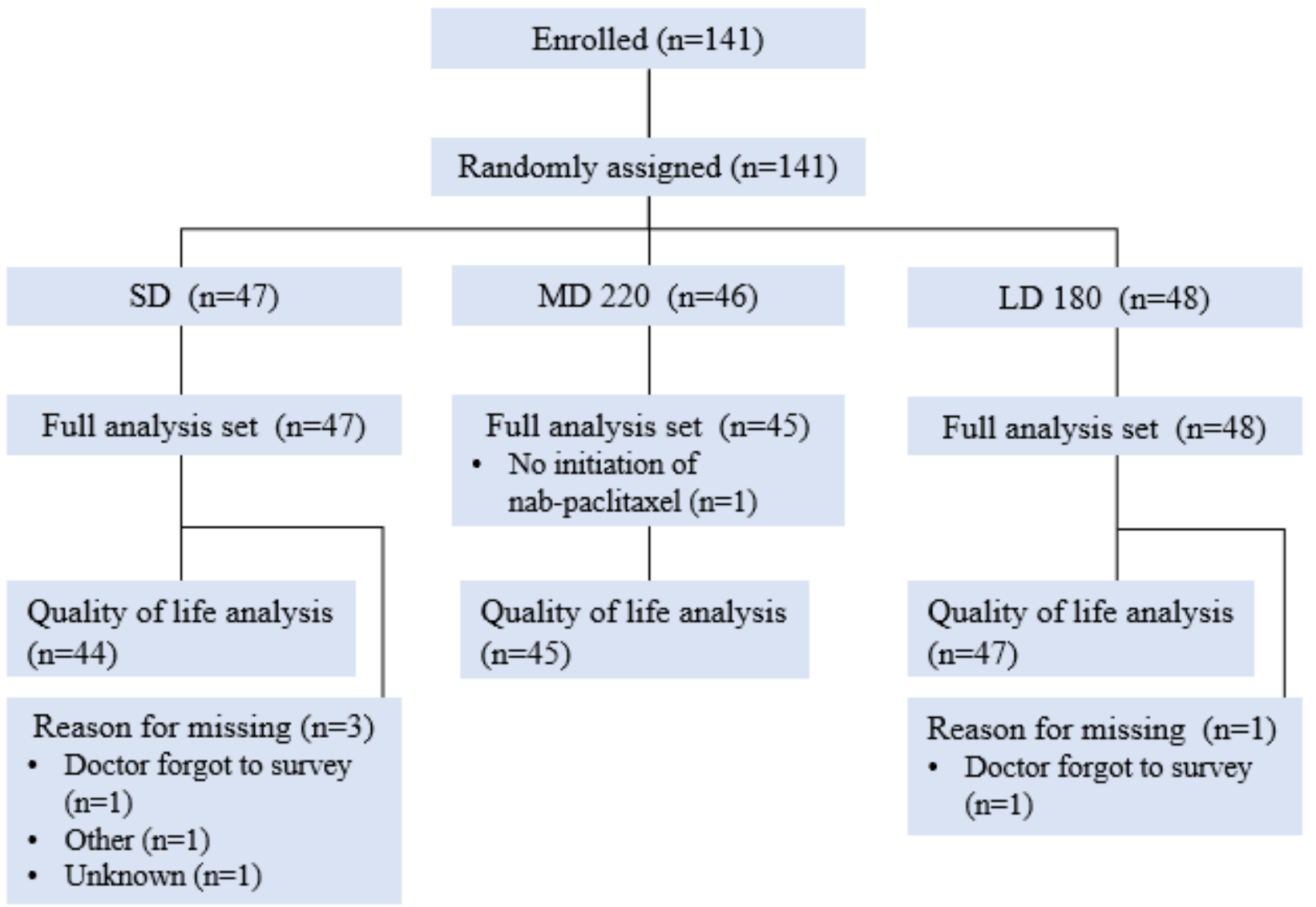

Figure 1

CONSORT diagram. 
A) FACT-Taxane TOI

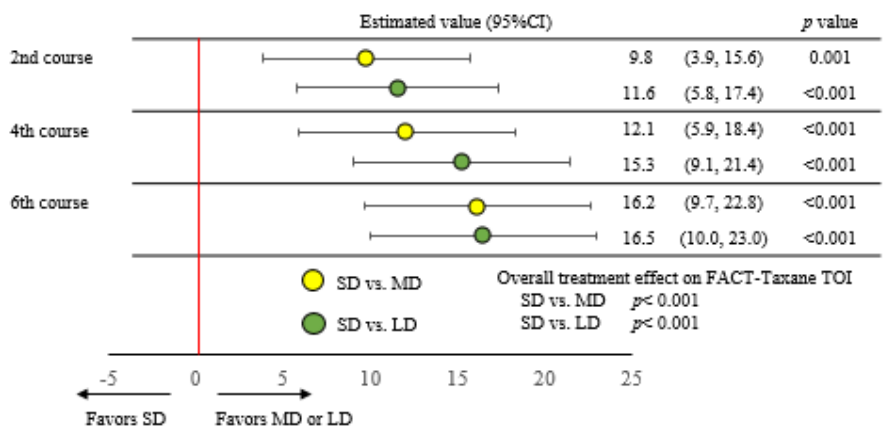

B) FACT-Taxane total

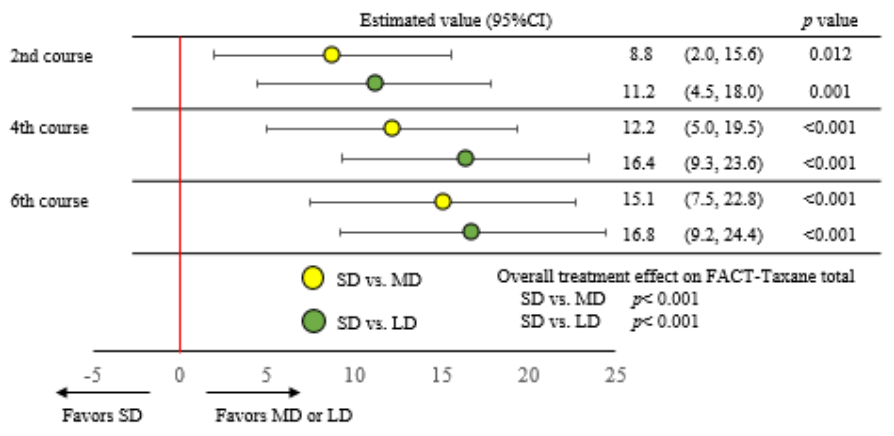

C) FACT-General total

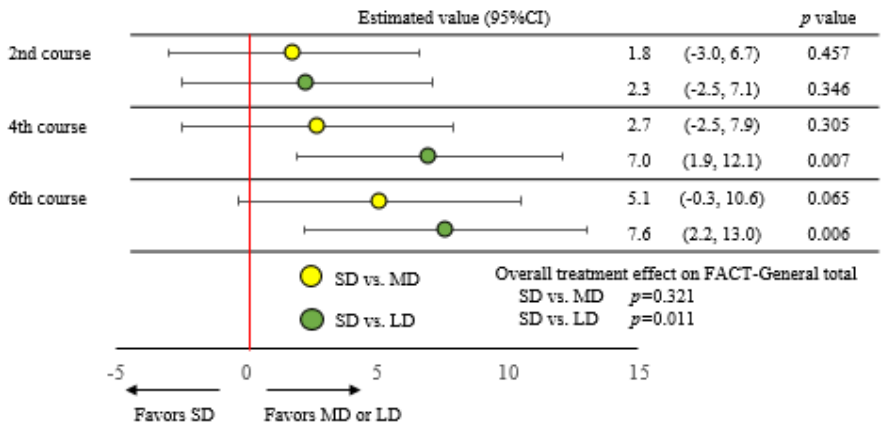

D) Taxane sub-scale

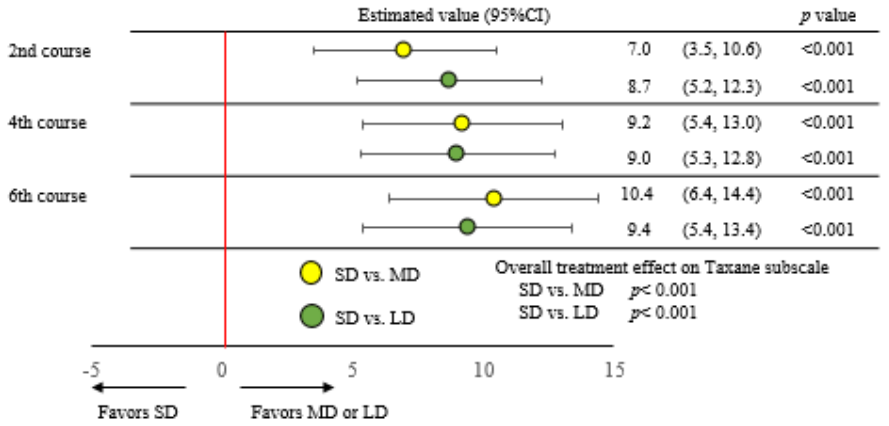

Figure 2

Estimates of mean differences and 95\% confidence intervals for changes in A) Functional Assessment of Cancer Therapy-Taxane trial outcome index (FACT-Taxane TOI), B) FACT-Taxane total, C) FACT-General total, and D) Taxane subscale scores, compared to SD $(260 \mathrm{mg} / \mathrm{m} 2$ of 3-week cycle nab-paclitaxel) in a mixed effects model for repeated measures. MD, $220 \mathrm{mg} / \mathrm{m} 2$ of 3-week cycle nab-paclitaxel; LD, 180 $\mathrm{mg} / \mathrm{m} 2$ of 3-week cycle nab-paclitaxel. 


\section{EQ-5D}

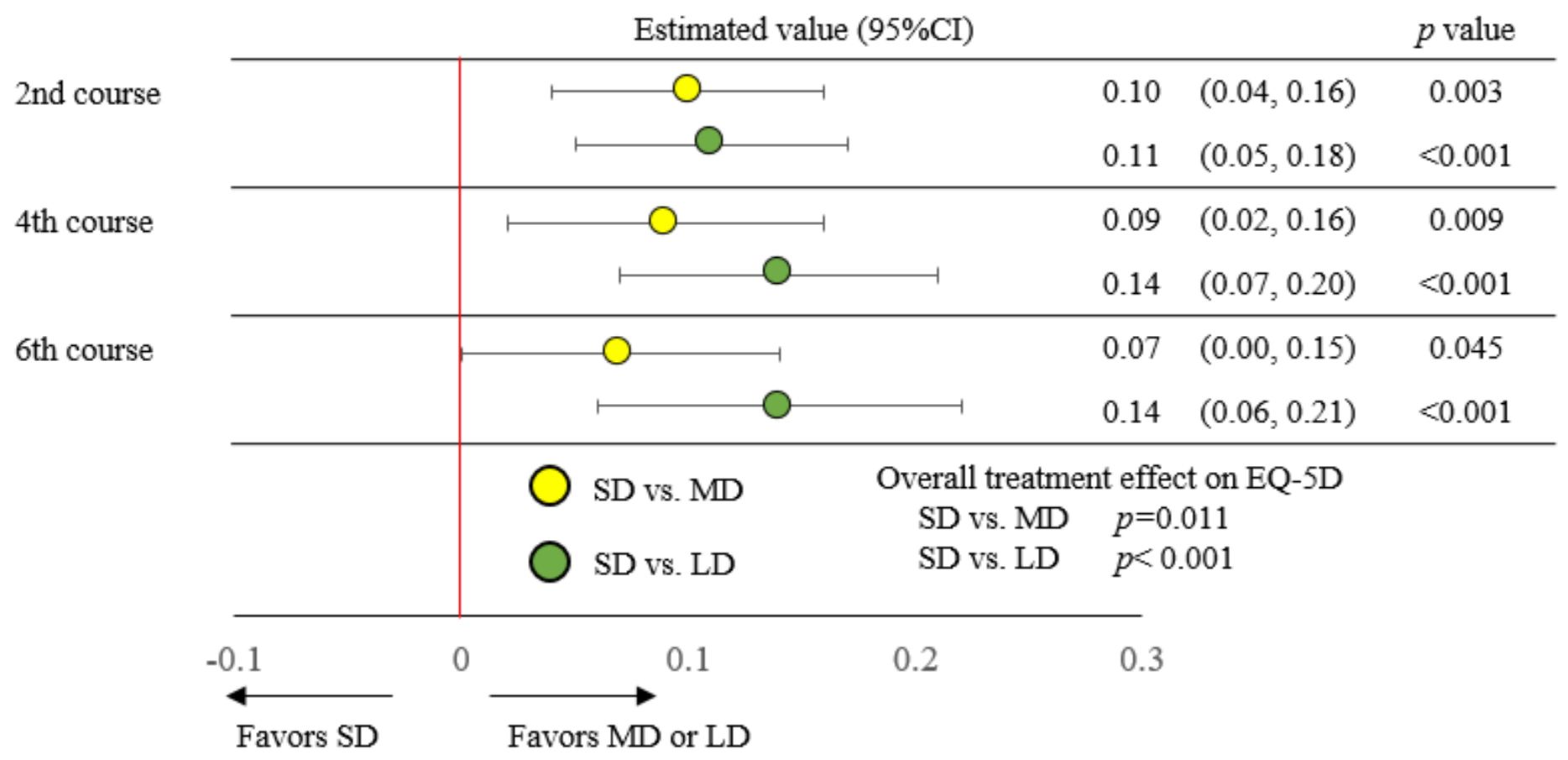

\section{Figure 3}

Estimates of mean differences and 95\% confidence intervals for changes in EuroQol 5-Dimension (EQ5D) scores, compared to SD ( $260 \mathrm{mg} / \mathrm{m} 2$ of 3-week cycle nab-paclitaxel) in a mixed effects model for repeated measures. MD, $220 \mathrm{mg} / \mathrm{m} 2$ of 3-week cycle nab-paclitaxel; LD, $180 \mathrm{mg} / \mathrm{m} 2$ of 3-week cycle nabpaclitaxel. 
A) CFS total

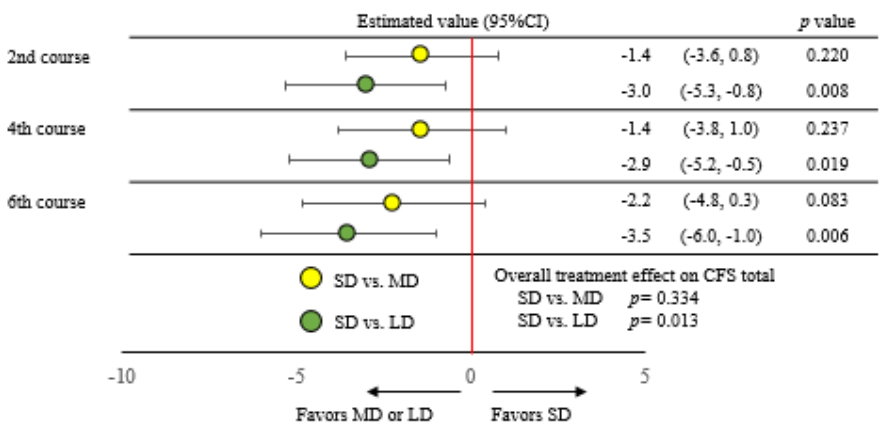

B) CFS physical

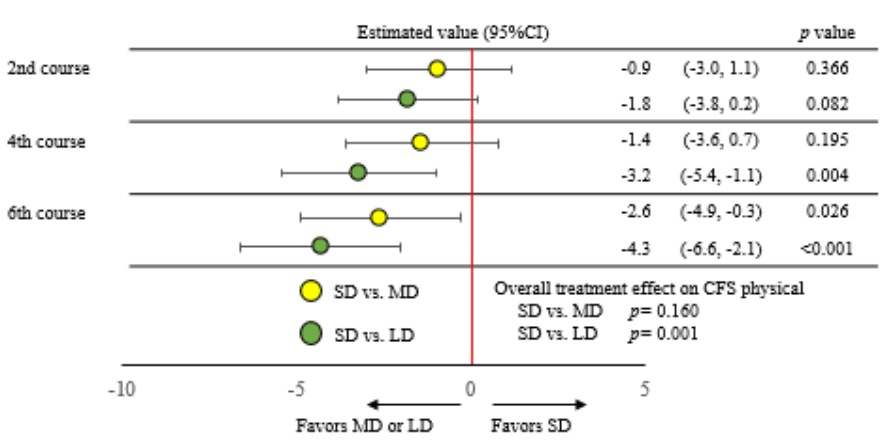

C) CFS affective
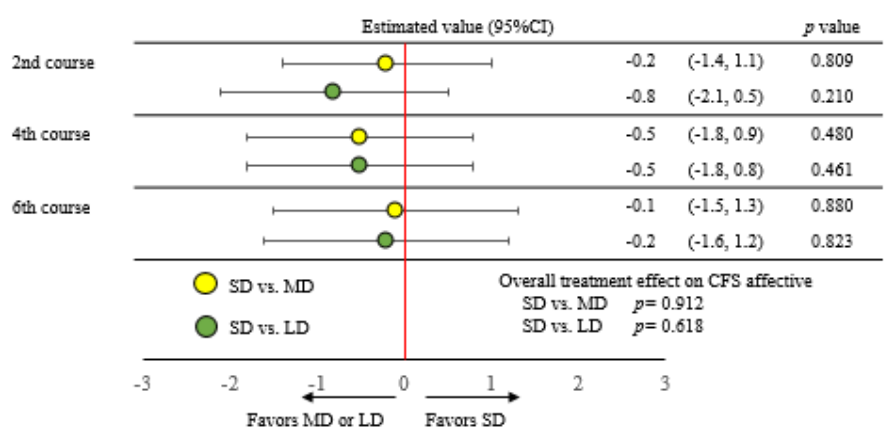

D) CFS cognitive

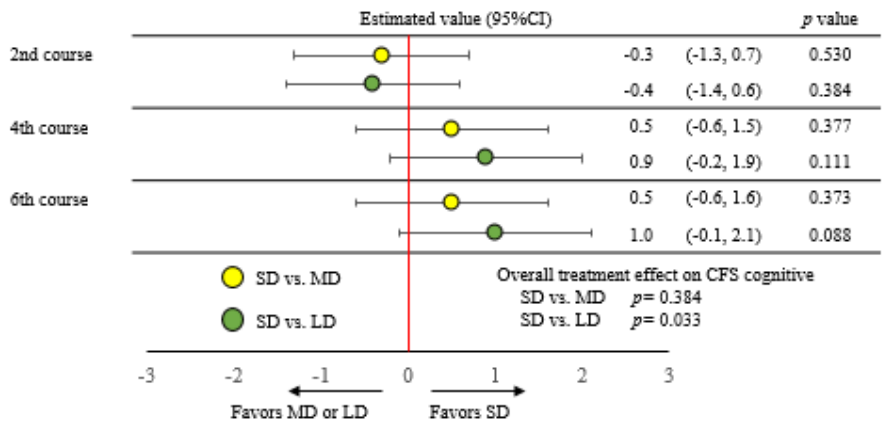

\section{Figure 4}

Estimates of mean changes and 95\% confidence interval for A) Cancer Fatigue Scale (CFS) total, B) physical, C) affective, and D) cognitive scores, compared to SD $(260 \mathrm{mg} / \mathrm{m} 2$ of 3-week cycle nabpaclitaxel) by mixed effects model for repeated measures. MD, $220 \mathrm{mg} / \mathrm{m} 2$ of 3-week cycle nabpaclitaxel; LD, $180 \mathrm{mg} / \mathrm{m} 2$ of 3-week cycle nab-paclitaxel.

\section{Supplementary Files}

This is a list of supplementary files associated with this preprint. Click to download.

- ABROADtrialQOLtabeleversion1.0.xlsx 